\title{
Incorporating molecular flexibility into three-dimensional structural kernels
}

\section{A Jahn}

Address: University of Tübingen, Sand 1, 72076 Tübingen, Germany from 4th German Conference on Chemoinformatics

Goslar, Germany. 9-II November 2008

Published: 5 June 2009

Chemistry Central Journal 2009, 3(Suppl I):OII doi:I0.II86/I752-I53X-3-SI-OII

This abstract is available from: http://www.journal.chemistrycentral.com/content/3/SI/OII

(C) 2009 Jahn; licensee BioMed Central Ltd.

The molecular conformation has an important impact on the biological activity of a ligand. To include the information of the conformational space into a similarity measure, it is necessary to perform a time-consuming conformational sampling of the structures. We approached this problem by introducing a new method to incorporate the geometry in a structured similarity measure. The basic concept is to breakdown the complete conformational space of a structure into local conformations of neighbour atoms with respect to core atoms. These local conformations are the trajectories of the second and third degree neighbours and arise from rotatable bonds. The parameters of the trajectories can be used to expand the physicochemical descriptors of structured similarity measures. We extended the optimal assignment kernel with our procedure and used the resulting pseudo-kernel in support vector machines. To assess the influence of our extension, we compared the QSAR results of the original and extended version of the pseudo-kernel on 8 benchmark data sets. The incorporation of the local conformation information significantly improves the model quality on 5 out of 8 data sets. 